\title{
Economic Impact of TAVI under monitored local anesthesia in Japan. Single center study.
}

Euroanaesthesia 2017 ?

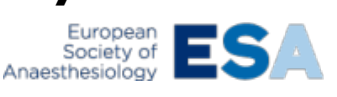

Ota T.', Nomura T. ${ }^{2}$, Nagamine K. ${ }^{3}$, Fukui K. ${ }^{3}$, Toyota K. ${ }^{3}$, Koide Y. ${ }^{3}$

IShonan Kamakura General Hospital, Dept of Anaesthesiology \& Intensive Care, Kamakura, Kanagawa, Japan, ${ }^{2}$ Yokohama City University, Dept of Anaesthesiology, Yokohama, Kanagawa, Japan, ${ }^{3}$ Shonan Kamakura General Hospital, Dept of Anaesthesiology, Kamakura, Japan

\section{Backgrounds:}

Trans-catheter aortic valve implantation (TAVI) has been undertaken worldwide as a surgical aortic valve replacement (SAVR) alternative surgery as a minimally invasive procedure. There are various opinions as to whether TAVI is under general anesthesia or under local anesthesia, but from the viewpoint of reducing the burden on patients and medical costs TAVI in local anesthesia is increasing, mainly in Europe and the United States.

As TAVI gets widespread in Japan, facilities to shift from general anesthesia (GA) to local anesthesia (LA) are beginning to be found.

This time we compared the general anesthesia and local anesthesia in our facility.

\section{Material \& Method:}

We examined TAVI cases (GA: 57 cases LA: 15 cases) retrospectively, during the period

from September 2015 to December 2016 at our hospital .

The primary endpoint was the staying time in the operating room,

the secondary evaluation items were the operating time, general anesthesia time.

Independent t-test was performed on the main evaluation items,

and comparison was made between the GA group and the LA group.

The staying time in the operating room is defined as from patient entry to exit.

This facility does not include PACU time because TAVI patients enter the ICU after completion of surgery.

The general anesthesia time is defined as the time from the start of oxygen administration to extubation.

In local anesthesia surgery the operation time is defined as synonymous with the anesthesia time.

There is no difference in procedures such as general anesthesia or disinfection after the start of sedation.

We excluded cases that changed to open heart surgery due to the occurrence of adverse events, and cases where data was insufficient.

\section{Result:}

The staying time in the operating room was II $3.8 \pm 32.7 \mathrm{~min}$ in the GA group,

$103.1 \pm 35.7 \mathrm{~min}$ in the LA group $(\mathrm{P}=0.27)$, and no significant difference was observed.

The operation time was $57.4 \pm 22.3 \mathrm{~min}$ in the GA group and $59.2 \pm 29.9 \mathrm{~min}$ in the LA group $(p=0.80)$, and no significant difference was observed.

The general anesthesia time was $87.6 \pm 30.6 \mathrm{~min}$.

\section{Assessment \& Discussion:}

According to the intention of the attending physician at the institution, TAVI operation was changed from September 2016 to LA in all cases.

In Japan, either TAVI is performed by general anesthesia or local anesthesia,

a very large cost difference (GA: $¥ 91,800-\approx € 740$, LA: $¥ 1,500-\approx € 12$ ) arises

from management according to its insurance system.

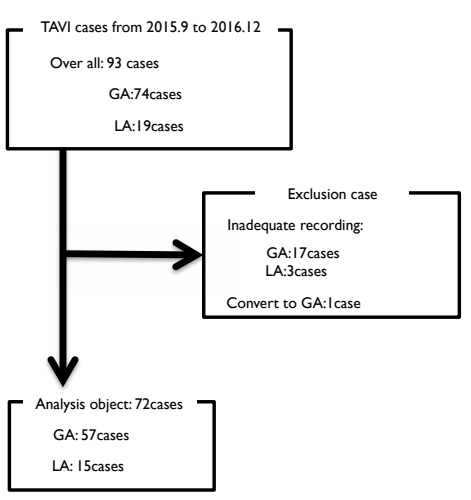

Also, from the national character of the Japanese and the characteristics of medical treatment, treatment under sedation or general anesthesia is preferred for surgery.

Therefore, TAVI tends to be preferred in Japan under general anesthesia.

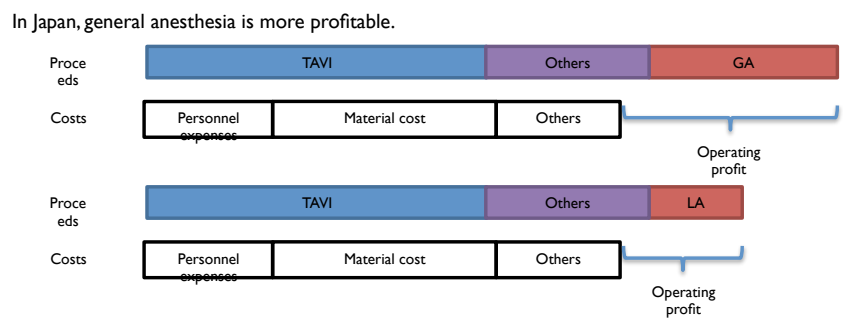

\begin{tabular}{llll} 
& GA & LA & $\mathrm{P}$ \\
\hline OR stay time & $113.8 \pm 32.7 \mathrm{~min}$ & $103.1 \pm 35.7 \mathrm{~min}$ & $\mathrm{p}=0.27$ \\
$\begin{array}{l}\text { Procedure } \\
\text { time }\end{array}$ & $57.4 \pm 22.3 \mathrm{~min}$ & $59.2 \pm 29.9 \mathrm{~min}$ & $\mathrm{p}=0.80$ \\
GA time & $87.6 \pm 30.6 \mathrm{~min}$ & $\mathrm{NA}$ &
\end{tabular}

PARTNER 2 trial: $\quad$ Anesthesia time: $207 \mathrm{~min} \quad$ Procedure time: $103 \mathrm{~min}$

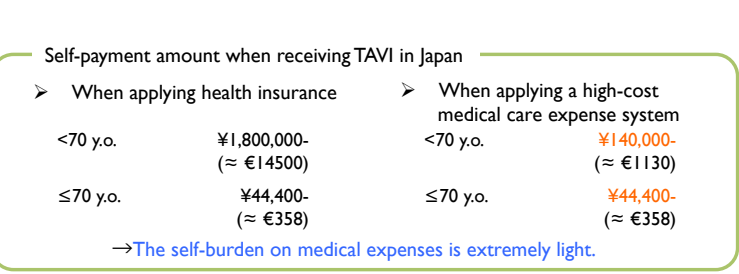

\footnotetext{
- Nationality of Japanese, characteristics of medical care

(1) Not good at making decisions

(2) Prefer surgery without consciousness

(3)Accept advanced medical treatment with cheap medical expenses

(4)Insert Public Expenses into Insurance Medical
}

But in general anesthesia,

(1)Physical stress such as cyclical fluctuation is strong for patients with severe aortic valve stenosis, which is an indication for TAVI

(2) Because it takes time from induction to awakening, turnover of the operating room is hindered and the number of operations can not be increased

So, TAVI under local anesthesia is expected to increase in Japan in the future.

In the data at our facility, there is no difference in staying time of the operating room and operating time between the GA group and the LA group

It seems that TAVI under general anesthesia has been optimized sufficiently.

If there is no difference in the operating room staying time and operating time due to the anesthesia method,

GA will be superior in terms of management as the cost cover by the insurance system is excellent.

There is no doubt that LA will be superior from the viewpoint of physical burden of patients and suppression of medical expenses,

In hospital management in the Japanese insurance system, there is a restriction that local anesthesia can not expect benefits that match medical resources to be introduced.

In order to be enforceable under "local anesthesia close to general anesthesia",

our facility is implementing TAVI under sedation / local anesthesia by Monitored Anesthesia Care (MAC).

As a result, TAVI with a light physical burden equivalent to local anesthesia can be enforced while receiving the same reimbursement as general anesthesia in the insurance system.

\section{Conclusion:}

TAVI under general anesthesia at the institution did not differ from that under local anesthesia in the operating room staying time.

TAVI under sedation / local anesthesia by Monitored Anesthesia Care (MAC) may be suitable for achieving compatibility between physical burden of patients in Japanese insurance system and cost management in hospital management. 